\title{
A Study of Fetal Sympatho-Vagal Balance at Various Gestational Periods Using the Length Transform on Magnetocardiographic Data
}

\author{
D Gutiérrez ${ }^{1}$, H Preissl $^{2,3}$, H Eswaran $^{3}$, CL Lowery $^{3}$ \\ ${ }^{1}$ Centro de Investigación y de Estudios Avanzados (CINVESTAV) Unidad Monterrey, N.L., México \\ ${ }^{2}$ MEG-Center, University of Tübingen, Tübingen, Germany \\ ${ }^{3}$ Department of Obstetrics and Gynecology, University of Arkansas for Medical Sciences, \\ Little Rock, AR, USA
}

\begin{abstract}
In this paper we characterize fetal sympatho-vagal balance by studying the correlation, time shifts, and ratios, between very-low-frequency (VLF), low-frequency $(L F)$, and high-frequency $(H F)$ components in heart rate (HR) variability over time. The HR is obtained from fetal magnetocardiographic (fMCG) recordings at various gestational periods. The frequency components are separated from the HR signal and pre-processed with the Length Transform (LT). The LT represents a temporarily smoothed time course of the data and has the property of highlighting the variability information of the signals over fixedlength windows. Therefore, we compute the correlations, time shifts, and ratios using the transformed data in order to avoid miscalculations and provide accurate quantitative measures. Our results show accordance with previous studies in sympathetic and parasympathetic control systems, and have the potential of characterizing fetal development.
\end{abstract}

\section{Introduction}

Recently, it has been of great interest to monitor the development of the fetus using magnetocardiographic techniques in order to characterize normal development as well as to identify possible pathologies [1],[2]. Many efforts have been placed in studying fetal heart rate (HR) variability, in particular time and frequency domain variables that are important markers for fetal well-being [3]-[5]. Assessments of fetal HR and fetal behavior are nowadays cornerstones of clinical fetal evaluation and neurodevelopmental research.

Fetal magnetocardiography (fMCG) is an excellent signal source for assessment of fetal HR, providing a precise signal that can be detected throughout the last half of preg- nancy and often earlier [6]. It is generally believed that clinical fetal HR monitors based on Doppler ultrasound lack the precision required to accurately assess beat-tobeat variations.

Although HR is affected by many factors, the utility of fetal HR monitoring is based on the dominant contribution of the autonomic nervous system. Absent the influence of nerves and hormones, the SA node will spontaneously discharge at a nearly constant rate. Parasympathetic, or vagal, tone causes HR to rise, while sympathetic tone causes HR to fall. Thus, HR is regulated primarily by balancing sympathetic and vagal tone [7].

Accurate quantification of the total vagal component in the HR is evidently vital for assessing the relative sympatho-vagal balance. Some studies suggest that very-low-frequency (VLF), low-frequency (LF) and highfrequency $(\mathrm{HF})$ oscillations characterizing the HR have some relationship with HR variability [8],[9]. However, there is not a reliable quantitative measure of such relationship yet. In [10] and [11], the ratio of the LF to HF components of the fetal HR is proposed as a measure of the sympatho-vagal balance, while in [9] the ratio of the VLF to LF is the one considered. These measures also rely on computing the correlations and time shifts between the different frequency components of the fetal HR. However, these calculations are very sensitive to signal-to-noise ratio of the data and often the results are not stable.

For this reason, we propose a method where the HR frequency components are analyzed after being transformed using the Length Transform (LT). This transformation highlights the variability information of the signals and makes the calculation of the correlation more precise. Therefore, we compute the corresponding correlations, time shifts, and ratios between the frequency components in the transformed domain. Section 2 describes all the processing steps required in our method. In Section 3 we show the applicability of our method through numeri- 
cal examples using real fMCG data, and in Section 4 we discuss the results and future work.

\section{Methods}

In this section we describe each of the steps involved in our proposed method for analyzing the HR data.

\subsection{Data acquisition}

fMCG measurements were performed with a 151channel SQUID system (SARA) [12]. SARA is a stationary, floor-mounted instrument where the mother sits and leans her abdomen against an anatomically shaped sensing surface. This design is inherently safe. The mother is comfortable and can gain easy access to or dismount from the system. The system is installed in a magnetically shielded room and equipped with high-order synthetic gradiometer noise cancellation, which effectively eliminates the vibrational noise transmitted by the mother. The array of SQUIDs covers the mother's anterior abdominal surface, from the perineum to the top of the uterus (in late gestation). The primary sensor flux transformers are axial first-order gradiometers with $8 \mathrm{~cm}$ baselines. The nominal SQUID noise density is $5 \mathrm{fT} / \sqrt{ } \mathrm{Hz}$. The primary sensor array is curved to fit the pregnant abdomen, covering a region of approximately $45 \mathrm{~cm}$ high and $33 \mathrm{~cm}$ wide, with an area of $1300 \mathrm{~cm}^{2}$ and inclined at $45^{\circ}$. The SARA system provides real-time access to fMCG measurements by means of a computational acquisition system that implements an on-line heart rate monitor [6].

\subsection{Extraction of frequency components}

In a similar way to [10], we implemented digital filters to extract the VLF, LF, and HF components of the HR signal. The HF component was extracted using a highpass butterworth filter of 5th order with a cutoff frequency of $0.2 \mathrm{~Hz}$. For the VLF component, we used a low-pass butterworth filter of 5 th order with a cutoff frequency of $0.05 \mathrm{~Hz}$. Finally, the LF component was obtained by subtracting the VLF and HF components from the original HR signal.

\subsection{Length transform}

Since we are interested in the variability information of the different frequency components, as well as on the correlation of the variability at different frequency bands, we propose to use the Length Transform (LT). The LT has proven to enhance short-term correlations and signal variability when used in bioelectrical signals [13]. Hence, let us denote the signal as $x_{(\cdot)}(n)$ for $n=1,2, \ldots, N$ time samples, where $(\cdot)$ indicates either the VLF, LF, or HF component (i.e. $x_{\mathrm{VLF}}, x_{\mathrm{LF}}$, or $x_{\mathrm{HF}}$ ). Then, the LT is given by [14]

$$
l_{(\cdot)}(n)=\sum_{k=n}^{n+q-1}\left|x_{(\cdot)}(k)-x_{(\cdot)}(k-1)\right|
$$

where $q$ is the length of the processing window. The LT represents a temporarily smoothed time course of the data, and can be interpreted as a measure of the path's length taken by signal to go from $x_{(\cdot)}(1)$ to $x_{(\cdot)}(N)$. Such length is proportional to the variability of the signal. Note that equation (1) corresponds to a single-channel transformation. However, the LT can also be applied for multichannel processing. The LT has been found to be superior to conventional transforms for feature extraction, and has been successfully applied in the problem of QRS detection for electrocardiographic data [14].

Under these conditions, we compute the corresponding transforms $l_{\mathrm{VLF}}(n), l_{\mathrm{LF}}(n)$ and $l_{\mathrm{HF}}(n)$ using (1) for each of the HR signals. For the value of $q$, we chose the number of samples (according to the sampling frequency of the signal) corresponding to 4 seconds in order to represent vagal activity.

\subsection{Correlation, delays, and ratios}

Previous reports (see [11]) have established that there exists a correlation between $l_{\mathrm{LF}}(n)$ and $l_{\mathrm{HF}}(n)$. Such correlation is maximum for $l_{\mathrm{HF}}\left(n-\tau_{\mathrm{HF}}\right)$, where $\tau_{\mathrm{HF}}$ is the delay of $l_{\mathrm{HF}}(n)$ with respect to $l_{\mathrm{LF}}(n)$. In order to obtain the value of $\tau_{\mathrm{HF}}$, we compute the correlation coefficients of $l_{\mathrm{LF}}(n)$ to $l_{\mathrm{HF}}\left(n-\tau_{0}\right)$, where $\tau_{0}$ varies from 0 to the number of samples corresponding to 20 seconds. Then, $\tau_{\mathrm{HF}}$ will be determined by value of $\tau_{0}$ for which the average correlation is maximum.

Once $\tau_{\mathrm{HF}}$ has been determined, we compute the ratio $r_{\mathrm{LF}-\mathrm{HF}}$ as

$$
r_{\mathrm{LF}-\mathrm{HF}}(n)=\frac{l_{\mathrm{LF}}(n)}{l_{\mathrm{HF}}\left(n-\tau_{\mathrm{HF}}\right)},
$$

and its mean value $\overline{r_{\text {LF-HF }}}$ as

$$
\overline{r_{\mathrm{LF}-\mathrm{HF}}}=\frac{1}{N} \sum_{n=1}^{N} r_{\mathrm{LF}-\mathrm{HF}}(n) .
$$

Changes in $\overline{r_{\mathrm{LF}-\mathrm{HF}}}$ at different gestation periods might be indicative of sympatho-vagal balance [10]. For this reason, $\overline{r_{\mathrm{LF}-\mathrm{HF}}}$ is a variable of interest in our analysis.

Other studies show that a correlation exists between the VLF and LF components, and the corresponding ratio between these components is the one indicating sympathovagal balance instead of $r_{\text {LF-HF }}$ [9]. Therefore, we compute also the correlation between $l_{\mathrm{VLF}}(n)$ and $l_{\mathrm{LF}}(n)$, and determine the delay $\tau_{\mathrm{LF}}$ that maximizes such correlation. Then, 
in a similar way as in (2) and (3), we define the ratio $r_{\text {VLF-LF }}$ and its mean $\overline{r_{\mathrm{VLF}-\mathrm{LF}}}$ as

$$
r_{\mathrm{VLF}-\mathrm{LF}}(n)=\frac{l_{\mathrm{VLF}}(n)}{l_{\mathrm{LF}}\left(n-\tau_{\mathrm{LF}}\right)},
$$

and

$$
\overline{r_{\mathrm{VLF}-\mathrm{LF}}}=\frac{1}{N} \sum_{n=1}^{N} r_{\mathrm{VLF}-\mathrm{LF}}(n) .
$$

\section{Numerical examples}

We conducted a series of experiments in which we applied the processing steps described in Section 2 to real fMCG data from six healthy patients at various gestational stages (see Table 1). The data were collected at a sampling rate of $1250 \mathrm{~Hz}$ during 10 minutes intervals. An example of the data acquired for one patient is shown in Figure 1.

\begin{tabular}{|c|c|c|c|c|c|c|c|c|c|c|}
\hline \hline \multirow{2}{*}{$\begin{array}{c}\text { Patient } \\
(\text { label })\end{array}$} & \multicolumn{10}{|c|}{ Weeks in gestation } \\
\hline & 27 & 29 & 30 & 31 & 32 & 33 & 34 & 35 & 36 & 37 \\
\hline 1 & & & & $\bullet$ & & $\bullet$ & & & $\bullet$ & \\
\hline 2 & & & & & & $\bullet$ & & & & \\
\hline 50 & $\bullet$ & $\bullet$ & & $\bullet$ & & $\bullet$ & & $\bullet$ & & $\bullet$ \\
\hline 505 & & & & & & $\bullet$ & $\bullet$ & & $\bullet$ & \\
\hline 507 & & & & $\bullet$ & & $\bullet$ & & $\bullet$ & & $\bullet$ \\
\hline 526 & & & $\bullet$ & & $\bullet$ & & $\bullet$ & & $\bullet$ & \\
\hline \hline
\end{tabular}

Table 1. Gestation periods at which data were acquired.

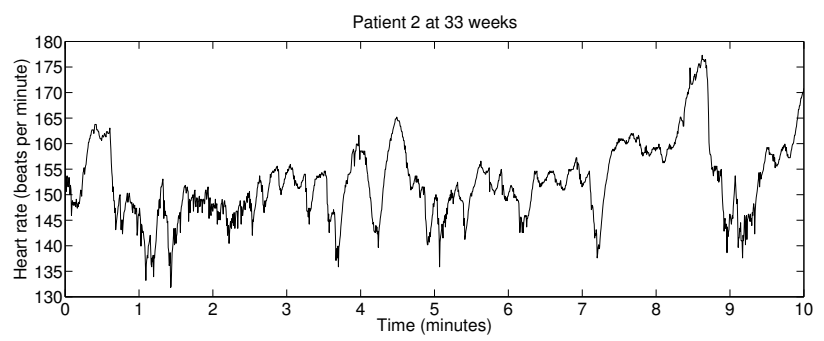

Figure 1. Heart rate of one patient as a function of time.

As result of processing the data of all subjects, we obtained a series of values of $\tau_{\mathrm{HF}}, \tau_{\mathrm{LF}}, \overline{r_{\mathrm{LF}-\mathrm{HF}}}$, and $\overline{r_{\mathrm{VLF}-\mathrm{LF}}}$ at different gestation periods. These results are shown in Figures 2 and 3.

Previous results (see [10]) reported high variations in the value of $\tau_{\mathrm{HF}}$. We believe those variations were product of miscalculations in the correlations. Instead, our results (see Figure 2) show less variability and are somehow clustered around a mean value of $1.36 \mathrm{~s}$ for $\tau_{\mathrm{HF}}$, while the mean value for $\tau_{\mathrm{LF}}$ turned to be $3.15 \mathrm{~s}$. If we consider that the sympatho-vagal balance is directly related to the delay between frequency components (which is known from
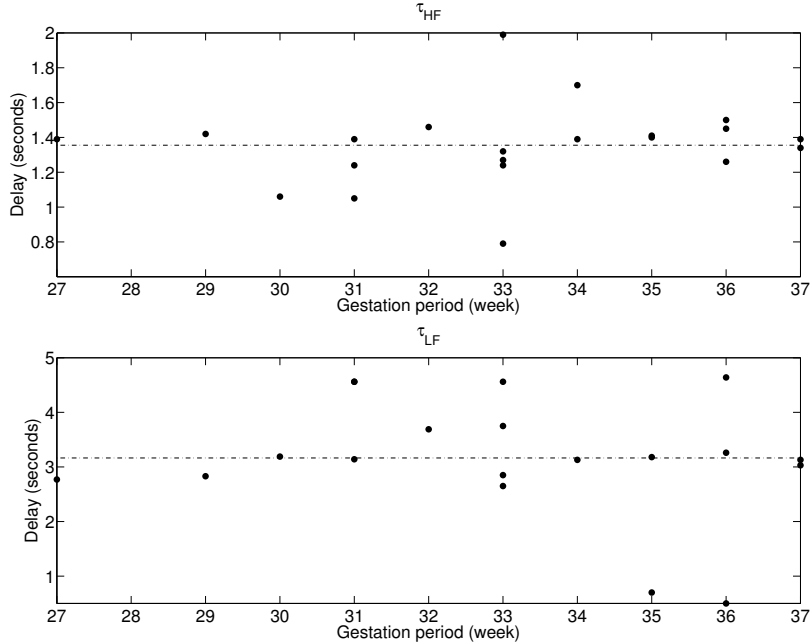

Figure 2. Values of $\tau_{\mathrm{HF}}$ and $\tau_{\mathrm{LF}}$ for all data in Table 1. The dash-dotted line indicates the mean value.
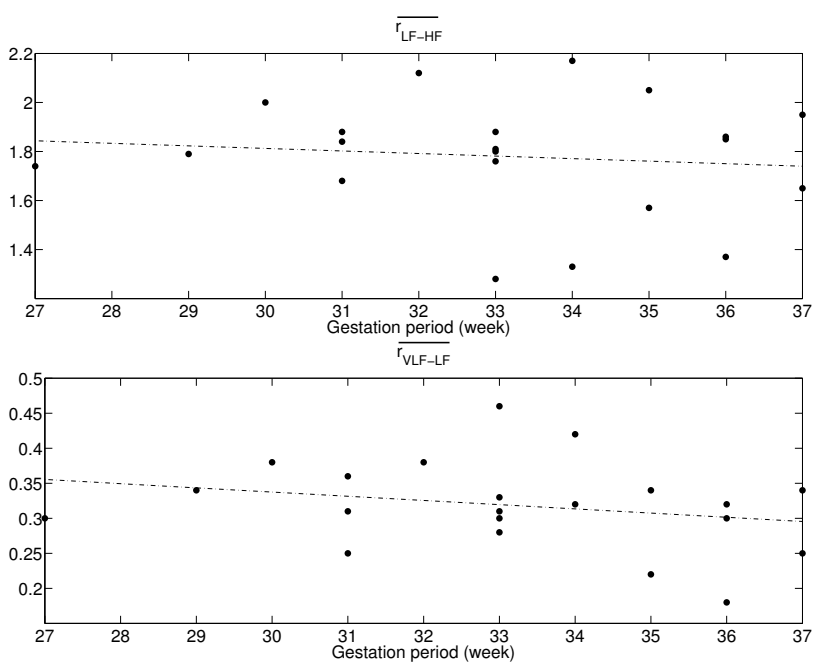

Figure 3. Values of $\overline{r_{\mathrm{LF}-\mathrm{HF}}}$ and $\overline{r_{\mathrm{VLF}-\mathrm{LF}}}$ for all data in Table 1. The dash-dotted line indicates the linear interpolation of the results.

[15] to be around 2 or $3 \mathrm{~s}$ ), then we can conclude that our results might be reflection of the sympatho-vagal balance, specially the result related to $\tau_{\mathrm{LF}}$ as it is closer to the known range.

In reference to $\overline{r_{\mathrm{LF}-\mathrm{HF}}}$, we also found a low variability, which is different to the results reported in [10]. Furthermore, we observed a small linear tendency in $\overline{r_{\mathrm{LF}-\mathrm{HF}}}$ to decrease at a rate of 0.01 times per week (see top graphic in Figure 3), which would translate into a reduction of 5.63\% in 10 weeks. Similar results were found for the case of $\overline{r_{\text {VLF-LF }}}$, which presented a decrease at a rate of 0.006 times per week (bottom graphic in Figure 3). However, this tendency is more significative for $\overline{r_{\mathrm{VLF}-\mathrm{LF}}}$ compared to $\overline{r_{\mathrm{LF}-\mathrm{HF}}}$ as it 
translates into a reduction of $16.9 \%$ in 10 weeks. These results are in accordance with those in [9] which suggest that $\overline{r_{\text {VLF-LF }}}$ is the ratio reflecting the sympatho-vagal balance, and it is reduced with fetal development. The decrease in $\overline{r_{\text {LF-HF }}}$ does not seem significative and might indicate that both the LF and the HF have a vagal component.

\section{Conclusions}

We presented a method to characterize sympatho-vagal balance based on fMCG recordings and the LT. This transformation highlights the variability information of the signal, which allows to reduce the errors in computing the correlation between different frequency components.

Our results show accordance with previous results suggesting that the ratio of VLF to LF is the one characterizing sympatho-vagal balance. We were not able to confirm a tendency of the delays to increase with respect to gestational period, as previously suggested. However, we still need to study this issue more comprehensively. Therefore, further sets of HR data recorded over extended periods need to be analyzed. Still, our results showed less variability thanks to the use of the LT.

Further work in this area will include more intensive application of our method to data in healthy and pathological conditions, as well as correlating the sympatho-vagal balance to measures of neural activity using fetal magnetoencephalography.

\section{Acknowledgment}

The authors would like to acknowledge the support of CTF Systems Inc., which is a subsidiary of VSM MedTech Ltd.

\section{References}

[1] van Leeuwen P, Halier B, Bader W, Geissler J, Trowitzsch $\mathrm{E}$, Gremeyer D. Magnetocardiography in the diagnosis of fetal arrhythmia. BJOG An International Journal of Obstetrics and Gynaecology 1999;106:1200-1208.

[2] Preissl H, Eswaran H, Murphy P, Lowery CL. The effect of fetal heart signals on fetal magnetoencephalogram recordings: a possible explanation for increased spectral power in case of intrauterine growth-retarded pregnancies. Prenatal Diagnosis 2003;23(7):603-605.

[3] Wakal RT. Assessment of fetal neurodevelopment via fetal magnetocardiography. Experimental Neurology 2004; 190(S1):65-71.

[4] Martin CB. Physiology and clinical use of fetal heart rate variability. Clinical Perinatology 1982;9:339-352.

[5] Karin J, Hirsch M, Akselrod S. An estimate of fetal autonomic state by spectral analysis of fetal heart rate fluctuations. Pediatric Research 1993;34:134-138.

[6] Waldert S, Bensch M, Bogdan M, Rosenstiel W, Schölkopf B, Lowery C, Eswaran H, Preissl H. Real-time fetal heart monitoring in biomagnetic measurements using adaptive real-time ICA. To appear in IEEE Transactions on Biomedical Engineering.

[7] Malliani A, Pagani M, Montano N, Mela GS. Sympathovagal balance: a reappraisal. Circulation 1998;98(23):2640 2643.

[8] Malliani A, Lombardi F, Pagani M. Power spectrum analysis of heart rate variability: a tool to explore neural regulatory mechanisms. Brithish Heart Journal 1994;71(1):1-2.

[9] David M, Hirsch M, Karin J, Toledo E, Akselrod S. An estimate of fetal autonomic state by time-frequency analysis of fetal heart rate variability. Journal of Applied Physiology 2007; 102:1057-1064.

[10] Zhuravlev YE, Rassi D, Mishin AA, Emery SJ. Dynamic analysis of beat-to-beat fetal heart rate variability recorded by squid magnetometer: quantification of sympatho-vagal balance. Early Human Development 2002;66(1):1-10.

[11] Rassi D, Mishin A, Zhuravlev YE, Matthes J. Time domain correlation analysis of heart rate variability in preterm neonates. Early Human Development 2005;81:341-350.

[12] Robinson SE, Burbank MB, Fife AA, Haid G, Kubik PR, Seachev I, Taylor B, Tillotson M, Vrba J, Wong G, Lowery CL, Eswaran H, Wilson JD, Murphy P, Preissl H. A biomagnetic instrument for human reproductive assessment. In Proceedings of the 12th International Conference in Biomagnetism. Espoo, Finland, August 2000; 919-922.

[13] Gutiérrez D, García-Nocetti F, Solano-González J. Classification of multichannel EEG data using lenght/energy transforms. In Proceedings of the 2005 1st IEEE International Workshop on Computational Advances in MultiSensor Adaptive Processing. Puerto Vallarta, Mexico, December 2005; 221-224.

[14] Gritzali F. Towards a generalized scheme for QRS detection in ECG waveforms. Signal Processing 1988;15(2):183192.

[15] Hill-Smith I, Purves RD. Synaptic delay in the heart: an ionophoretic study. Journal of Physiology 1978;279:31-54.

Address for correspondence:

David Gutiérrez, PhD.

CINVESTAV Unidad Monterrey

Cerro de las Mitras 2565, Col. Obispado, C.P. 64060, Monterrey,

N.L., México. Tel. +52-81-8220-1740.

E-mail: davidgtz@ cinvestav.mx

URL: http://hecate.mty.cinvestav.mx/ david 